\title{
Advanced search for the extension of unresolved TeV sources with H.E.S.S.
}

First measurement of the extension of the Crab nebula at TeV energies

\author{
M. Holler ${ }^{* a}$, D. Berge ${ }^{b}$, J. Hahn ${ }^{c}$, D. Khangulyan ${ }^{d}$, R. D. Parsons ${ }^{c}$, for the H.E.S.S. \\ collaboration \\ ${ }^{a}$ Institut für Astro- und Teilchenphysik, Universität Innsbruck \\ ${ }^{b}$ GRAPPA, Anton Pannekoek Institute for Astronomy, University of Amsterdam \\ ${ }^{c}$ Max-Planck-Institut für Kernphysik \\ ${ }^{d}$ Department of Physics, Rikkyo University \\ E-mail: markus.holler@uibk.ac.at
}

\begin{abstract}
The resolution power of current Imaging Atmospheric Cherenkov Telescopes is presently restricted to scales of a few arcminutes. In the very high-energy (VHE; $E>100 \mathrm{GeV}$ ) gamma-ray regime, the measurement of source sizes that are comparable to or smaller than the resolution of the instrument is usually limited by statistics and in particular by the uncertainties in the characterisation of the instrument Point Spread Function (PSF). The PSF varies strongly with observation and instrument conditions demanding time dependent simulations of these conditions. Employing such simulations, we substantially improve our understanding of the H.E.S.S. PSF and are now able to probe source extensions well below one arcminute scale. We present the results of this new approach applied to known VHE gamma-ray sources and show how this enables us to reveal for the first time the extension of the Crab nebula at $\mathrm{TeV}$ gamma-ray energies, with a width of $52^{\prime \prime}$ assuming a Gaussian source shape.
\end{abstract}

35th International Cosmic Ray Conference - ICRC2017

12-20 July, 2017

Bexco, Busan, Korea

* Speaker. 


\section{Instrument and Dataset}

H.E.S.S. is an array of five Imaging Atmospheric Cherenkov Telescopes (IACTs) located in the Khomas Highlands of Namibia. The Crab nebula has been observed with the H.E.S.S. telescopes since the beginning of operations in 2004. For the measurement presented here we only use observations with the four IACTs of the first phase of H.E.S.S. In addition to the standard runquality selection criteria [1], we apply additional cuts e.g. on the maximum wind speed $(<3 \mathrm{~m} / \mathrm{s})$, the observation wobble offset $\left(<0.8^{\circ}\right)$, and the zenith angle of the observations $\left(<55^{\circ}\right)$ to define a high-quality dataset for extension measurements. The resulting Crab nebula dataset consists of observations performed between February 2004 and November 2011, amounting to a total deadtimecorrected live time of $25.7 \mathrm{~h}$. Since the Crab nebula is a Northern source, the typical observation zenith angles at the H.E.S.S. site in the Southern hemisphere are $45^{\circ}-50^{\circ}$.

\section{Analysis and Results}

The data analysis is performed using semi-analytical air-shower templates [2]. To further improve the angular resolution, we use a tight cut on the direction reconstruction uncertainty for the event selection. Furthermore, we demand energies of $E>0.7 \mathrm{TeV}$ to eliminate potential systematic effects near the energy threshold of the dataset ${ }^{1}$. We detect the Crab nebula with a statistical significance of $137 \sigma$ (using Eq. 17 from [3]) and obtain a signal to background ratio of 58 within $0.1^{\circ}$ around the source position. We find a total number of about 4600 excess events, the majority of them with energies between the lower cut of 0.7 and $10 \mathrm{TeV}$. We show the resulting distribution of events as a histogram in squared angular distance $\left(\vartheta^{2}\right)$ in the top panel of Fig. 1. The value of $\vartheta^{2}$ is calculcated with respect to the centroid of the gamma-ray excess count distribution in the sky. The centroid position in equatorial coordinates (J2000) is $\alpha=5 \mathrm{~h} 34 \mathrm{~m} 30.9 \mathrm{~s} \pm(1.2 \mathrm{~s})_{\text {stat }} \pm(20 \mathrm{~s})_{\text {sys }}$, $\delta=+22^{\circ} 00^{\prime} 44.5^{\prime \prime} \pm 1.1_{\text {stat }}^{\prime \prime} \pm 20_{\text {sys }}^{\prime \prime}$ (systematic error from [4]), at a distance of $16.8^{\prime \prime}$ from, and within uncertainties compatible with, the Crab pulsar location.

Dedicated run-wise Monte-Carlo (MC) simulations of the dataset, including the actual instrument and observation conditions at the time the data were recorded, are generated as described in [5]. We re-weight the simulations to mimic the energy spectrum of the Crab nebula and analyse them with the same algorithms and analysis configurations as the actual data. The resulting $\vartheta^{2}$ histogram of this MC analysis serves as the PSF for this source and dataset and is shown in the upper panel of Fig. 1 . The $68 \%, 80 \%$, and $90 \%$ containment radii of our PSF are $0.05^{\circ}, 0.07^{\circ}$, and $0.09^{\circ}$, respectively.

As shown in Fig. 1, the PSF is highly inconsistent with the distribution of the gamma-ray excess counts. The residuals in the lower panel of Fig. 1 indicate a clear broadening of the data compared to the simulated PSF. To study this further, we perform a 2D morphology fit with Sherpa [6], using the sky images of gamma-ray-like events around the Crab nebula, of gamma-ray-like background events estimated from a ring well outside the source [7], as well as the simulated PSF. To determine the best fit, we convolve the PSF with a 2D Gaussian with different widths. For each width, we calculate a likelihood value to assess the compatibility of the data and the convolved

\footnotetext{
${ }^{1}$ The H.E.S.S. energy threshold for the Crab nebula is relatively large because the source is only visible under large zenith angles.
} 

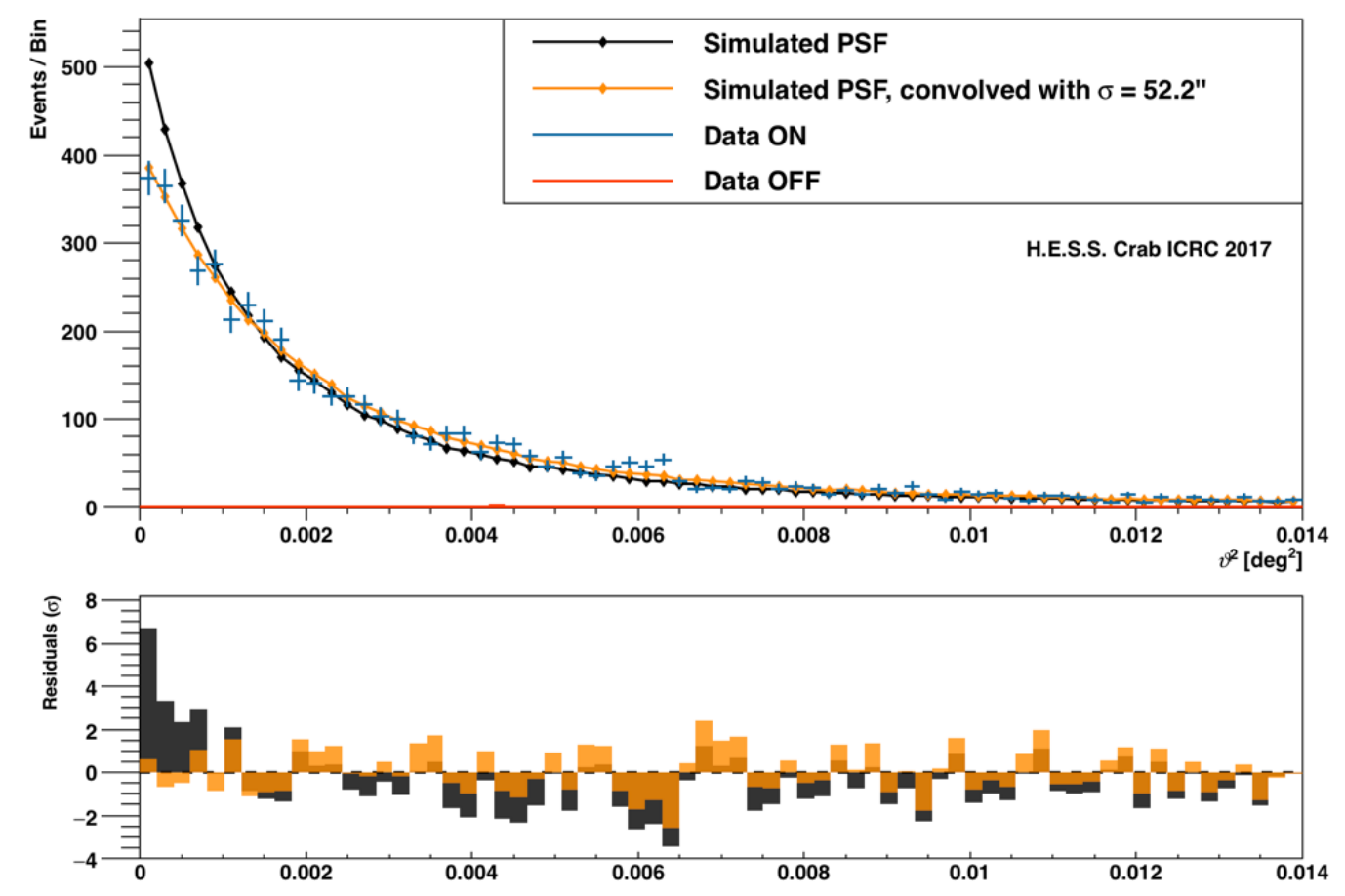

Figure 1: Top: $\vartheta^{2}$ histogram of gamma-ray-like events from the Crab nebula $(O N)$ along with background events from displaced sky regions ( $O F F$, scaled to the $O N$ region). The background events are barely visible in the plot due to the large $S / B$ ratio. For comparison, the simulated PSF and the PSF convolved with the best-fit Gaussian are shown as well. Bottom: Significance of the bin-wise deviation $(M C-d a t a)$ of the measured excess when compared to the plain PSF (black) and the convolved one (orange).

PSF. We find the best-fit extension to be $\sigma_{2 \mathrm{D}, \mathrm{G}}=52.2^{\prime \prime} \pm 2.9_{\mathrm{stat}}^{\prime \prime} \pm 7.8_{\mathrm{sys}}^{\prime \prime}$, with a preference of an extension of the Crab nebula over a point-source assumption of $\mathrm{TS} \approx 83^{2}$. As systematic uncertainty of the extension we quote the quadratic sum of uncertainties related to the calibration and analysis method, to the spectral shape used to re-weight the MC PSF, and to the fit method.

The resulting best-fit convolution is also plotted in Fig. 1. It clearly provides a good description of the data both in the upper panel and the residuals in the lower panel.

To demonstrate the robustness of our results, we apply the same analysis using time-dependent simulations to two other bright and highly significant extragalactic gamma-ray sources, the active galactic nuclei PKS 2155-304 and Markarian 421. As illustrated in Fig. 2, we find both sources compatible with being point-like and show upper limits on their extension. In both cases, these limits are well below the measured extension of the Crab nebula. We note that Markarian 421 culminates at large zenith angles of $\theta>60^{\circ}$ at the H.E.S.S. site, making this source a particularly convincing test of our PSF understanding: even under such challenging observation conditions, Markarian 421 appears to be point-like. As we also show in Fig. 2, we tested the Crab nebula dataset for a zenith angle dependence by splitting the observations in two datasets above and below $46^{\circ}$. The measured extensions are compatible with each other.

Also shown in Fig. 2 is the extension of the Crab nebula cross-checked with an independent

\footnotetext{
${ }^{2} \mathrm{TS}$ is the likelihood ratio test statistic and $\sqrt{\mathrm{TS}}$ can be interpreted as statistical significance.
} 


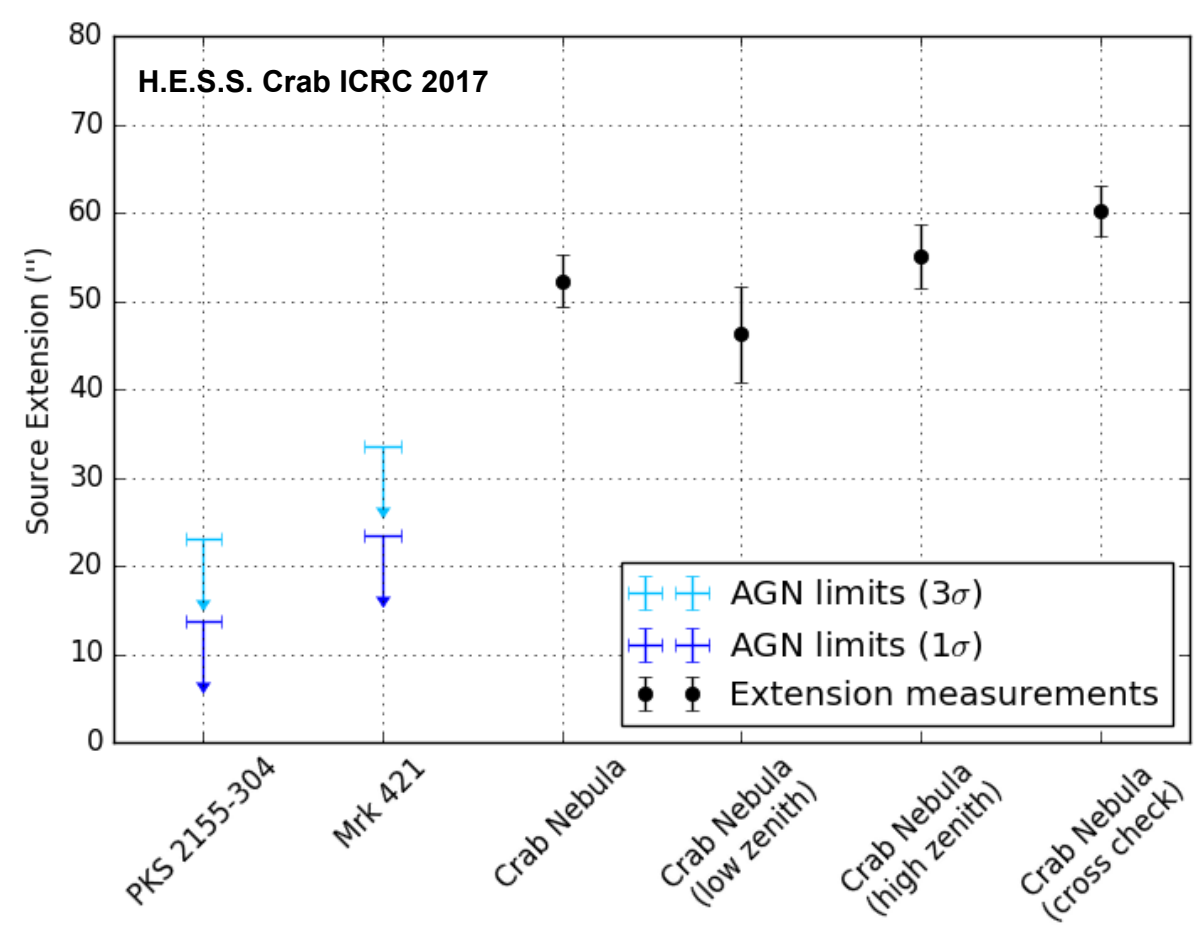

Figure 2: Overview of the derived extension upper limits of PKS 2155-304 and Markarian 421, as well as the measured extension of the Crab nebula and systematic checks. The low and high zenith angle band correspond to $44-46^{\circ}$ and $46-55^{\circ}$, respectively.

calibration, reconstruction, and analysis method [8]. We find this second extension measurement slightly larger than our nominal value, and incorporate the difference as one of our systematic uncertainties mentioned above.

Our VHE gamma-ray extension of the Crab nebula is compared to the morphology found at UV wavelengths and X-ray energies in the left and right panel of Fig. 3, respectively. The H.E.S.S. extension covers a good fraction of the optical nebula. Comparing the $\mathrm{TeV}$ gamma-ray emission from Inverse Compton scattering to the $\mathrm{keV}$ synchrotron X-ray emission, we find that the nebula measured with H.E.S.S. is significantly larger than when measured with Chandra. This result is naturally explained by the radiation cooling of electrons. The Crab nebula size therefore decreases with increasing electron energy, and the energies of electrons producing the $\mathrm{TeV}$ gamma rays are well below those of electrons emitting the hard X-rays measured by Chandra. The measured size of the $\mathrm{TeV}$ gamma-ray nebula can be reproduced within the standard magnetohydrodynamic model of Kennel and Coroniti $[12,13]$ assuming a magnetisation parameter $\sigma \approx 0.01$.

\section{Conclusions}

Here we document the ability of the H.E.S.S. IACT array to robustly measure VHE gammaray source extensions down to $30-40^{\prime \prime}$. The performance boost provided by using time-dependent simulations allows us to resolve for the first time the Inverse Compton component of the Crab nebula. The emission region size we find is well below the previously most constraining upper 

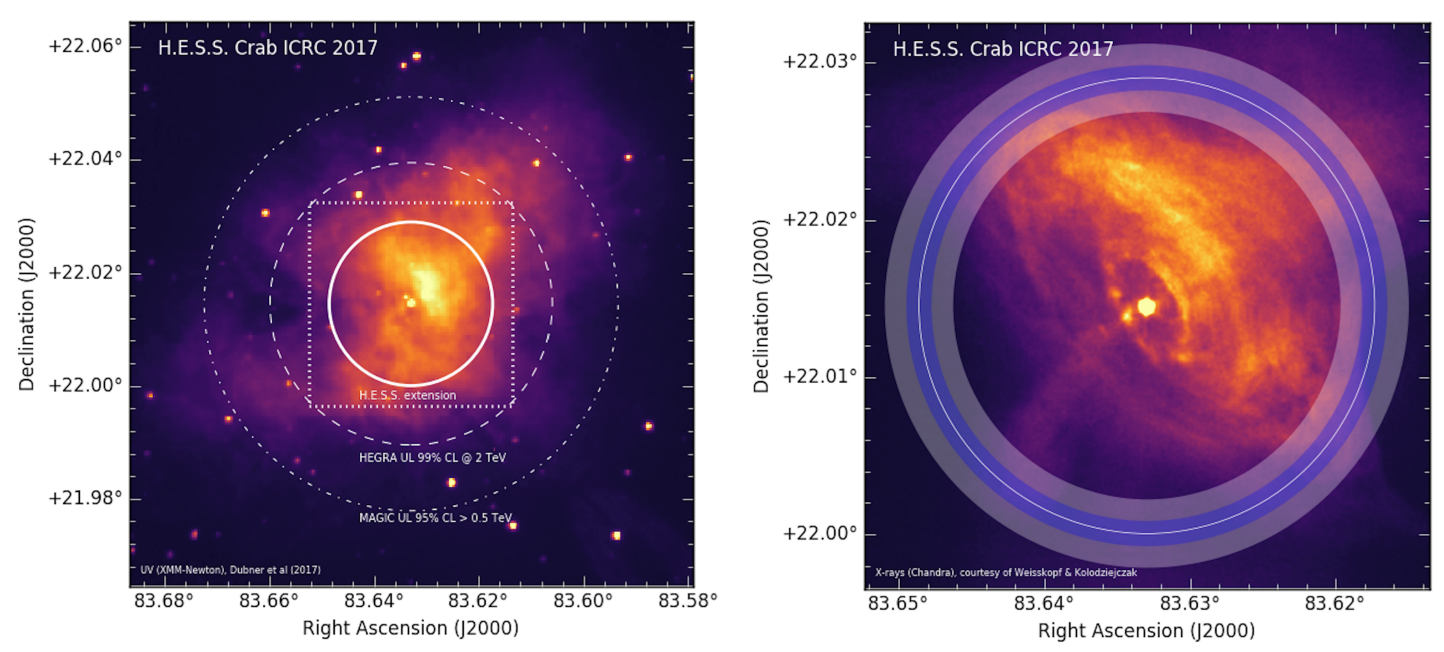

Figure 3: Left: UV $(\lambda=291 \mathrm{~nm})$ image of the Crab nebula [9]. The MAGIC and HEGRA extension upper limits of $2.2^{\prime}$ [10] and $1.5^{\prime}$ [11] are drawn as dash-dotted and dashed lines, respectively. The extent of the sky region of the right panel is indicated as dotted square, and the H.E.S.S. extension (Gaussian $\sigma$ as given in the main text) is drawn as a solid circle. All circles are centred on the Crab pulsar position. Right: Chandra $\mathrm{X}$-ray image of the Crab nebula. The H.E.S.S. extension is shown as solid white circle overlaid on top of shaded annuli indicating the statistical and systematic uncertainties of our measurement.

limit of $1.5^{\prime}[11]$ and is determined with a high accuracy of $\approx 15 \%$. Compared to the synchrotron Crab nebula seen in keV X-rays, the VHE gamma-ray emission region is clearly more extended.

\section{Acknowledgements}

We gratefully acknowledge the support from the agencies and institutions listed here.

\section{References}

[1] F. A. Aharonian et al., Observations of the Crab nebula with HESS, Astronomy \& Astrophysics 457 (Oct., 2006) 899-915, [astro-ph/0607333].

[2] M. de Naurois and L. Rolland, A high performance likelihood reconstruction of $\gamma$-rays for imaging atmospheric Cherenkov telescopes, Astroparticle Physics 32 (2009), no. 5231 - 252, [arXiv:0907.2610].

[3] T.-P. Li and Y.-Q. Ma, Analysis methods for results in gamma-ray astronomy, The Astrophysical Journal 272 (Sept., 1983) 317-324.

[4] S. Gillesen, Sub-Bogenminuten-genaue Positionen von TeV-Quellen mit H.E.S.S. Dissertation, Ruprecht-Karls-Universität Heidelberg, Heidelberg, Germany, 2004.

[5] M. Holler et al., Run-Wise Simulations for Imaging Atmospheric Cherenkov Telescope Arrays, POS (ICRC2017) 755, 2017.

[6] P. Freeman, S. Doe, and A. Siemiginowska, Sherpa: a mission-independent data analysis application, in Astronomical Data Analysis (J.-L. Starck and F. D. Murtagh, eds.), vol. 4477 of Proceedings of SPIE, pp. 76-87, Nov., 2001. astro-ph/0108426. 
[7] D. Berge, S. Funk, and J. Hinton, Background modelling in very-high-energy $\gamma$-ray astronomy, Astronomy \& Astrophysics 466 (May, 2007) 1219-1229, [astro-ph / 0610959 ].

[8] R. D. Parsons and J. A. Hinton, A Monte Carlo template based analysis for air-Cherenkov arrays, Astroparticle Physics 56 (Apr., 2014) 26-34, [arXiv: 1403.2993 ].

[9] G. Dubner et al., Morphological Properties of the Crab Nebula: A Detailed Multiwavelength Study Based on New VLA, HST, Chandra, and XMM-Newton Images, The Astrophysical Journal 840 (May, 2017) 82, [arXiv:1704.0296].

[10] J. Albert et al., VHE $\gamma$-Ray Observation of the Crab Nebula and its Pulsar with the MAGIC Telescope, The Astrophysical Journal 674 (Feb., 2008) 1037-1055, [arXiv: 0705.3244 ].

[11] F. A. Aharonian et al., Optimizing the angular resolution of the HEGRA telescope system to study the emission region of VHE gamma rays in the Crab Nebula, Astronomy \& Astrophysics 361 (Sept., 2000) 1073-1078.

[12] C. F. Kennel and F. V. Coroniti, Confinement of the Crab pulsar's wind by its supernova remnant, The Astrophysical Journal 283 (Aug., 1984) 694-709.

[13] C. F. Kennel and F. V. Coroniti, Magnetohydrodynamic model of Crab nebula radiation, The Astrophysical Journal 283 (Aug., 1984) 710-730. 